\title{
A canonical form for a symplectic involution
}

\author{
Harry W. Braden ${ }^{1}$
}

Received: 5 January 2018 / Revised: 5 April 2018 / Accepted: 5 April 2018 /

Published online: 21 May 2018

(C) The Author(s) 2018

\begin{abstract}
We present a canonical form for a symplectic involution $S \in \operatorname{Sp}(2 g, \mathbb{Z})$, $S^{2}=\mathrm{Id}$; the construction is algorithmic. Application is made in the Riemann surface setting.
\end{abstract}

Keywords Canonical form $\cdot$ Symplectic involution

Mathematics Subject Classification $15 \mathrm{~A} 21 \cdot 14 \mathrm{H} 37 \cdot 14 \mathrm{Q} 05$

\section{Introduction}

Canonical forms for matrices with integer coefficients are useful in many settings: one may think of the Smith Normal Form or Frobenius's decomposition of a skew matrix [15], both of which will be used later in the paper. In his study of real abelian varieties Comessatti $[5,6]$ introduced a canonical form for an involution in $\operatorname{GL}(n, \mathbb{Z})$ (the precise result will be recalled later). Here we shall establish the symplectic analogue of Comessatti's theorem providing a canonical form for a symplectic involution, $S \in \mathrm{Sp}(2 g, \mathbb{Z})=\left\{\gamma \in \mathrm{GL}(2 g, \mathbb{Z}) \mid \gamma^{T} J \gamma=J\right\}$, where throughout $J=\left(\begin{array}{cc}0 & 1 g \\ -1 g & 0\end{array}\right)$ is the canonical symplectic pairing. The canonical form with an immediate corollary is given by:

Theorem 1.1 Let $S \in \operatorname{Sp}(2 g, \mathbb{Z})$ be a symplectic involution, $S^{T} J S=J$ and $S^{2}=\mathrm{Id}$. Then $S$ is symplectically equivalent to one of the form $S=\left(\begin{array}{ll}a & 0 \\ 0 & a\end{array}\right)$ where

$凶$ Harry W. Braden

hwb@ed.ac.uk

1 Maxwell Institute and School of Mathematics, The University of Edinburgh, James Clerk Maxwell Building, Peter Guthrie Tait Road, Edinburgh EH9 3FD, UK 


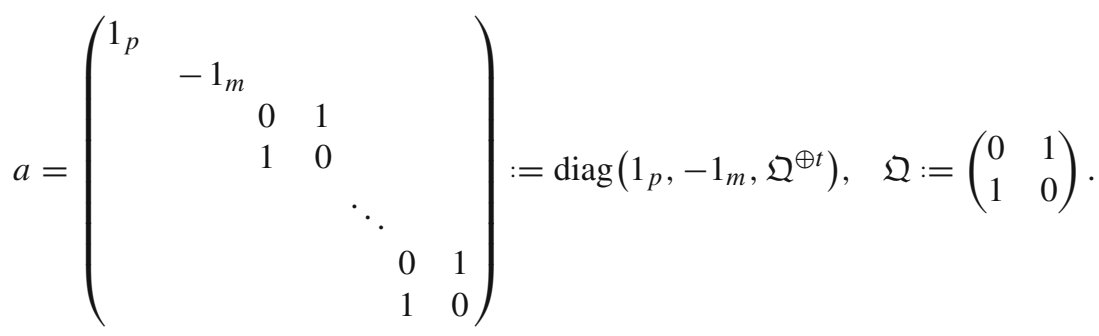

If $t$ is the number of $2 \times 2$ blocks $\mathfrak{Q}$ then $g=p+m+2 t$.

Corollary 1.2 Let $S$ be a symplectic involution of $W=\mathbb{Z}^{2 g}$ with canonical pairing. Then $W=L_{1} \oplus L_{2},\left\langle L_{i}, L_{i}\right\rangle=0$, with stable Lagrangian subspaces $S L_{i}=L_{i}$.

Some special cases of Theorem 1.1 are known in the context of Riemann surfaces. The canonical form yields a different proof of

Theorem 1.3 Let $S$ be a conformal involution of the Riemann surface $\mathcal{C}$ of genus $g$ with $k$ fixed points and let $\mathrm{C}^{\prime}=\mathcal{C} /\langle S\rangle$ be the quotient surface of genus $g^{\prime}$. We may find a homology basis for $\mathcal{C}$ in which $S$ takes the form (1) where $g=p+m+2 t$, $g^{\prime}=p+t$ and either

- $k>0$ and $p=0$ whence $g^{\prime}=t$ and $m=k / 2-1$,

- $k=0, p=1$ whence $g^{\prime}=t+1$ and $m=0$.

The former case yields a result of Gilman [12] while the latter yields Fay's example of an unbranched covers [11, Chapter IV].

We note that the proof we present of Theorem 1.1 is constructive. Before turning to the proofs we will give some further background including Comessatti's result that we will employ. If one could find a module-theoretic proof of Corollary 1.2 then Theorem 1.1 would follow from Comessatti's theorem.

Finally we remark that the theorems described in this paper are of significant utility in the computational study of Riemann surfaces. Relevant for this volume Edge often studied curves and geometric configurations with high symmetry such as Klein's curve [8], Bring's curve [10] and the Fricke-Macbeath curve [9]. The first and third of these curves are Hurwitz surfaces whose automorphism groups (Hurwitz groups) are quotients of the (2, 3, 7)-triangle group possessing the symplectic involutions of the theorem. Bring's curve with automorphism group $S_{5}$ is a quotient of the $(2,4,5)$-triangle group [17]. Using the theorems outlined in this paper for curves with symmetries adapted homology bases may be found that, for example, significantly simplify the period matrices and the calculation of the vector of Riemann constants $[2,3]$.

\section{Background}

In order to place the result in context its helpful to see the parallel between several results for the general linear and symplectic groups. First, 
Lemma 2.1 If $\operatorname{gcd}\left(x_{1}, \ldots, x_{m}\right)=d$ then there exists $U \in \operatorname{GL}(m, \mathbb{Z})$ such that

$$
\left(x_{1}, \ldots, x_{m}\right) U=(d, 0, \ldots, 0) .
$$

Lemma 2.2 If $\operatorname{gcd}\left(x_{1}, x_{2}, \ldots, x_{2 g}\right)=d$ then there exists $S \in \operatorname{Sp}(2 g, \mathbb{Z})$ such that

$$
\left(x_{1}, x_{2}, \ldots, x_{2 g}\right) S=(d, 0, \ldots, 0) \text {. }
$$

Lemma 2.1 is classical (see for example [15]). Lemma 2.2 seems less well-known; the first proof of this I am aware of is [16].

In his study of real abelian varieties Comessatti [5,6] introduced the following canonical form.

Theorem 2.3 Let $M$ be a free $\mathbb{Z}$-module of rank $m$ and let $S \in$ Aut $(M)$ be an involution. Let rank $M_{ \pm}:=s_{ \pm}$where $M_{ \pm}$are the submodules

$$
M_{+}:=\{x \in M \mid S x=x\}, \quad M_{-}:=\{x \in M \mid S x=-x\} .
$$

Then we may find a basis of $M$ such that $S$ takes the form

$$
S=\operatorname{diag}\left(1_{s_{+}-\lambda},-1_{s_{-}-\lambda}, \mathfrak{Q}^{\oplus \lambda}\right)
$$

where we have $\lambda$ copies of the matrix $\mathfrak{Q}=\left(\begin{array}{ll}0 & 1 \\ 1 & 0\end{array}\right)$. Moreover, $s_{+}, s_{-}$and $\lambda$ are invariants of $S$.

Here $\lambda$ is known as the Comessatti character (a modern review of Comessatti's work may be found in [4]). Silhol [18] expressed these invariants in terms of the group cohomology of $G=\langle 1, S\rangle$. Then

$$
H^{i}(G, M)= \begin{cases}M_{+} \cong\left(\mathbb{Z}_{2}\right)^{s^{+}} & i=0, \\ \frac{M_{-}}{(1-S) M} \cong\left(\mathbb{Z}_{2}\right)^{s^{-}-\lambda} & i \equiv 1 \bmod 2, \\ \frac{M_{+}}{(1+S) M} \cong\left(\mathbb{Z}_{2}\right)^{s^{+}-\lambda} & i \equiv 0 \bmod 2, \quad i>0 .\end{cases}
$$

In the study of real structures the focus of attention are anti-holomorphic involutions $S^{T} J S=-J, S^{2}=$ Id rather than holomorphic involutions. Comessatti showed that an anti-holomorphic involution $S$ takes the form $\left(\begin{array}{cc}1_{g} & H \\ 0 & -1_{g}\end{array}\right)$ where $H$ is a symmetric bilinear form over $\mathbb{Z}_{2}$. These forms are determined by the rank of $H$ and whether $\operatorname{diag}(H)$ is nonzero or not. We have either (see for example [19])

$$
H=\operatorname{diag}\left(\mathfrak{Q}^{\oplus r}, 0^{\oplus g-2 r}\right) \quad \text { or } \quad H=\operatorname{diag}\left(1^{\oplus r}, 0^{\oplus g-r}\right) .
$$

An algorithm that constructs such a basis for a Riemann surface with real structure is given in [14]. 
Remark 2.4 Comessatti's theorem admits both purely module-theoretic and constructive proofs. The proof that follows of Theorem 1.1 is constructive. If one could find a module-theoretic proof of Corollary 1.2 then the theorem would follow from Comessatti's theorem. A generalisation of Comessatti's theorem exists for all cyclic groups of prime order [7, Theorem 74.3] though the corresponding generalisation of Theorem 1.1 has yet to be established.

\section{Proof of Theorem 1.1}

The proof is constructive. Writing $S=\left(\begin{array}{ll}a & b \\ c & d\end{array}\right)$ where $a, b, c, d$ are block $g \times g$ integer matrices, the constraints $S^{T} J S=J$ and $S^{2}=$ Id mean that $S$ takes the form

$$
S=\left(\begin{array}{cc}
a & b \\
c & a^{T}
\end{array}\right), \quad 0=b+b^{T}=c+c^{T}=a b+b a^{T}=c a+a^{T} c, a^{2}+b c=\mathrm{Id} .
$$

We remark that if $U \in \mathrm{GL}(g, \mathbb{Z})$ and $\mu=\mu^{T}$ then the rotations and translations

$$
R_{U}=\left(\begin{array}{cc}
U & 0 \\
0 & U^{-1}
\end{array}\right), \quad T_{\mu}=\left(\begin{array}{ll}
1 & \mu \\
0 & 1
\end{array}\right)
$$

are symplectic. In particular the similarity transformation

$$
R_{U^{-1}} S R_{U}=\left(\begin{array}{cc}
U^{-1} a U & U^{-1} b U^{-1 T} \\
U^{T} c U & U^{T} a^{T} U^{-1 T}
\end{array}\right)
$$

yields a similarity transformation on $a$ and takes $c$ to a congruent matrix.

The first step of the proof is to make a symplectic transformation so that $c=0$. The Frobenius decomposition of the skew matrix $c$ says there exists $U \in \operatorname{GL}(g, \mathbb{Z})$ such that $c=U^{T} D U$ where the congruent matrix $D$ takes the form

$$
D=\operatorname{diag}\left(d_{1} \mathfrak{J}, d_{2} \mathfrak{J}, \ldots, d_{s} \mathfrak{J}, 0^{\oplus g-2 r}\right), \quad \mathfrak{J}:=\left(\begin{array}{cc}
0 & 1 \\
-1 & 0
\end{array}\right),
$$

with $d_{i} \mid d_{i+1}$ for $1 \leqslant i \leqslant s-1$ and rank $c=2 s$. By using the appropriate symplectic transformation $R_{U}$ we may suppose that $c$ is in the Frobenius form $D$ stated. Let $\operatorname{gcd}\left(a_{21}, d_{1}\right)=v$. Then there are $p, q, u, v \in \mathbb{Z}$ such that

$$
a_{21}=v p, \quad d_{1}=v q, \quad u p-v q=1 .
$$

Then the symplectic matrix 


$$
T=\left(\begin{array}{cccccc}
1 & 0 & 0 & 0 & 0 & 0 \\
0 & u & 0 & 0 & v & 0 \\
0 & 0 & 1_{g-2} & 0 & 0 & 0 \\
0 & 0 & 0 & 1 & 0 & 0 \\
0 & q & 0 & 0 & p & 0 \\
0 & 0 & 0 & 0 & 0 & 1_{g-2}
\end{array}\right)
$$

is such that

$$
\text { T.S. } T^{-1}=\left(\begin{array}{cc}
a^{\prime} & b^{\prime} \\
D^{\prime} & a^{\prime}
\end{array}\right), \quad D^{\prime}=\operatorname{diag}\left(0 \times \mathfrak{J}, d_{2} \mathfrak{J}, \ldots, d_{s} \mathfrak{J}, 0^{\oplus g-2 r}\right) .
$$

Continuing in this way we see that $S$ is similar via a symplectic transformation to the case when $c=0$.

With $c=0$ we see from from (2) that $a^{2}=1$. Using the freedom to make a similarity transform to $a$, noted above, we may now use Comessatti's Theorem 2.3 to put $a$ into the canonical form

$$
a=\operatorname{diag}\left(1_{r},-1_{s}, \mathfrak{Q}^{\oplus l}\right)
$$

for appropriate $r$ and $s$. At this stage we have that

$$
S=\left(\begin{array}{cc}
a & b \\
0 & a^{T}
\end{array}\right), \quad 0=b+b^{T}=a b+b a^{T}
$$

and in block form

$$
a=\left(\begin{array}{lll}
1_{r} & & \\
& -1_{s} & \\
& & Q
\end{array}\right), \quad Q=\operatorname{diag}\left(\mathfrak{Q}^{\oplus l}\right)
$$

where $Q$ is a $2 l \times 2 l$ matrix. Now solving for $0=b+b^{T}=a b+b a^{T}$ we find that $b$ has the form

$$
b=\left(\begin{array}{ccc}
0 & x & y \\
-x^{T} & 0 & z \\
-y^{T} & -z^{T} & \gamma
\end{array}\right), \quad \gamma+\gamma^{T}=0=\gamma Q+Q \gamma, \quad y=-y Q, \quad z=z Q .
$$

Here $x \in M_{r, s}(\mathbb{Z}), y \in M_{r, 2 l}(\mathbb{Z}), z \in M_{s, 2 l}(\mathbb{Z}), \gamma \in M_{2 l, 2 l}(\mathbb{Z})$. Thus each row of the matrix $y$ takes the form

$$
\left(y_{i 1},-y_{i 1}, y_{i 2},-y_{i 2}, \ldots, y_{i l},-y_{i l}\right), \quad 1 \leqslant i \leqslant r,
$$

while each row of the matrix $z$ takes the form

$$
\left(z_{j 1}, z_{j 1}, z_{j 2}, z_{j 2}, \ldots, z_{j l}, z_{j l}\right), \quad 1 \leqslant j \leqslant s .
$$


Further the skew-symmetric matrix $\gamma$ may be written as $2 \times 2$ blocks

$$
\gamma=\left(\begin{array}{cccc}
d_{11} & m_{12} & \cdots & m_{1 l} \\
-m_{12}^{T} & d_{22} & & \\
\vdots & & \ddots & \\
-m_{1 l}^{T} & & & d_{l l}
\end{array}\right), \quad d_{i i}=\left(\begin{array}{cc}
0 & \alpha_{i} \\
-\alpha_{i} & 0
\end{array}\right), \quad m_{i j}=\left(\begin{array}{cc}
\beta_{i j} & \delta_{i j} \\
-\delta_{i j}-\beta_{i j}
\end{array}\right)
$$

Observe that

$$
T_{\mu} S T_{-\mu}=\left(\begin{array}{cc}
a & b+\mu a^{T}-a \mu \\
0 & a^{T}
\end{array}\right)
$$

and so if

$$
\mu=\left(\begin{array}{lll}
\mu_{1} & \mu_{2} & \mu_{3} \\
\mu_{2}^{T} & \mu_{4} & \mu_{5} \\
\mu_{3}^{T} & \mu_{5}^{T} & \mu_{6}
\end{array}\right), \quad \mu_{1}=\mu_{1}^{T}, \quad \mu_{4}=\mu_{4}^{T}, \quad \mu_{6}=\mu_{6}^{T}
$$

then $\mu=\mu^{T}$ and

$$
\mu a^{T}-a \mu=\left(\begin{array}{ccc}
0 & -2 \mu_{2} & \mu_{3} Q-\mu_{3} \\
2 \mu_{2}^{T} & 0 & \mu_{5} Q+\mu_{5} \\
\mu_{3}^{T}-Q \mu_{3}^{T} & -\mu_{5}^{T}-Q \mu_{5}^{T} & \mu_{6} Q-Q \mu_{6}
\end{array}\right)
$$

Thus if we choose the rows of the matrix $\mu_{3}$ to be $\left(y_{i 1}, 0, y_{i 2}, 0, \ldots, y_{i l}, 0\right), 1 \leqslant i \leqslant r$, then

$$
y+\mu_{3} Q-\mu_{3}=0 .
$$

Similarly if the rows of the matrix of the matrix $\mu_{5}$ to be $-\left(z_{j 1}, 0, z_{j 2}, 0, \ldots, z_{j l}, 0\right)$, $1 \leqslant j \leqslant s$, then

$$
z+\mu_{5} Q+\mu_{5}=0
$$

Finally taking $\mu_{6}$ to be of the form

$$
\mu_{6}=\left(\begin{array}{cccc}
d_{11}^{\prime} & m_{12}^{\prime} & \cdots & m_{1 l}^{\prime} \\
m_{12}^{\prime T} & d_{22}^{\prime} & & \\
\vdots & & \ddots & \\
m_{1 l}^{\prime T} & & & d_{l l}^{\prime}
\end{array}\right), \quad d_{i i}^{\prime}=\left(\begin{array}{cc}
-\alpha_{i} & 0 \\
0 & 0
\end{array}\right), \quad m_{i j}^{\prime}=\left(\begin{array}{cc}
-\delta_{i j}-\beta_{i j} \\
0 & 0
\end{array}\right)
$$

yields

$$
\gamma+\mu_{6} Q-Q \mu_{6}=0
$$


Therefore we may take $b$ to be of the form form

$$
b=\left(\begin{array}{ccc}
0 & x & 0 \\
-x^{T} & 0 & 0 \\
0 & 0 & 0
\end{array}\right)
$$

and where $x$ is a $(0,1)$-matrix.

At this stage we have shown that we may choose a symplectic basis in which the involution $S$ takes the block form

$$
S=\left(\begin{array}{cccccc}
1_{r} & 0 & 0 & 0 & x & 0 \\
0 & -1_{s} & 0 & -x^{T} & 0 & 0 \\
0 & 0 & Q & 0 & 0 & 0 \\
0 & 0 & 0 & 1_{r} & 0 & 0 \\
0 & 0 & 0 & 0 & -1_{s} & 0 \\
0 & 0 & 0 & 0 & 0 & Q
\end{array}\right)
$$

where $x$ is a $(0,1)$-matrix. Further, by use of the rotation $R_{U}$ with $U$ of the form

$$
U^{-1}=\left(\begin{array}{ccc}
A & & \\
& B & \\
& & 1
\end{array}\right), \quad A \in \mathrm{GL}(r, \mathbb{Z}), \quad B \in \mathrm{GL}(s, \mathbb{Z}),
$$

we may transform $x$ to $A x B^{T}$. Making use of the Smith normal form and the ability to remove even integral parts of $x$ by a translation we may therefore assume $x$ to have only 1's and 0's along the diagonal and be zero off the diagonal. Suppose there are $t \leqslant \min (r, s) 1$ 's on the diagonal. Then we may write

$$
S=\left(\begin{array}{cccccc}
1_{r-t} & 0 & 0 & & & \\
0 & -1_{s-t} & 0 & & & \\
0 & 0 & Q & & & \\
& & & 1_{r-t} & 0 & 0 \\
& & & 0 & -1_{s-t} & 0 \\
& & & 0 & 0 & Q
\end{array}\right) \oplus S^{\prime} \oplus \cdots \oplus S^{\prime}
$$

where we have $t$ copies of the symplectic matrix

$$
S^{\prime}=\left(\begin{array}{cccc}
1 & 0 & 0 & 1 \\
0 & -1 & -1 & 0 \\
0 & 0 & 1 & 0 \\
0 & 0 & 0 & -1
\end{array}\right)
$$

and we are indicating a symplectic decomposition. Now consider 


$$
V=\left(\begin{array}{cccc}
1 & 0 & 0 & 0 \\
1 & 0 & 0 & 1 \\
0 & 1 & 1 & 0 \\
0 & -1 & 0 & 0
\end{array}\right)
$$

Then

$$
V^{T}\left(\begin{array}{cc}
0 & 1_{2} \\
-1_{2} & 0
\end{array}\right) V=\left(\begin{array}{cc}
0 & 1_{2} \\
-1_{2} & 0
\end{array}\right), \quad V S^{\prime} V^{-1}=\left(\begin{array}{cccc}
0 & 1 & 0 & 0 \\
1 & 0 & 0 & 0 \\
0 & 0 & 0 & 1 \\
0 & 0 & 1 & 0
\end{array}\right)
$$

Thus by conjugation we may bring $S$ to the desired form and have established the theorem.

\section{Proof of Theorem 1.3}

We now apply our results in the setting where we have a Riemann surface $\mathcal{C}$ of genus $g>0$ with nontrivial finite group of symmetries $G \leqslant$ Aut $\mathcal{C}$ (Aut $\mathcal{C}$ is necessarily finite for $g \geqslant 2)$. Aut $\mathcal{C}$ acts naturally on $\mathcal{C}, H_{1}(\mathcal{C}, \mathbb{Z})$ and the harmonic differentials. Consider the quotient Riemann surface $\pi: \mathcal{C} \rightarrow \mathcal{C}^{\prime}=\mathcal{C} / G$ of genus $g^{\prime}$. From (1) $g=(p+t)+(m+t)$ and we can form $p+t$ invariant differentials and $m+t$ anti-invariant differentials under the action of $S$; then $g^{\prime}=p+t$ is the genus of $\mathrm{C}^{\prime}$. By Riemann-Hurwitz if there are $k \geqslant 0$ fixed points of $S$ then $g-1=2\left(g^{\prime}-1\right)+k / 2$ yields

$$
m=p+\frac{k}{2}-1
$$

Hurwitz showed that $\phi \in$ Aut $\mathcal{C}$ is the identity if and only if it induces the identity on $H_{1}(\mathcal{C}, \mathbb{Z})$. Accola [1] strengthened this result and showed that for $g \geqslant 2$ if there exist two pairs of canonical cycles such that (in homology) $\phi\left(\mathfrak{a}_{1}\right)=\mathfrak{a}_{1}, \phi\left(\mathfrak{a}_{2}\right)=\mathfrak{a}_{2}$, $\phi\left(\mathfrak{b}_{1}\right)=\mathfrak{b}_{1}$ and $\phi\left(\mathfrak{b}_{2}\right)=\mathfrak{b}_{2}$ then $\phi$ is the identity (simpler proofs of this result were obtained by Earle as well as Grothendieck and Serre, see [13]). Accola's result means in the canonical form for the symplectic involution above we have $p \leqslant 1$. We will have therefore proven the theorem once we establish

Lemma 4.1 If $k>0$ then $p=0$.

Proof Let $\left\{\gamma_{a}\right\}_{a=1}^{2 g}$ be a basis for $H_{1}(\mathcal{C}, \mathbb{Z})$ ordered so that $\gamma_{a}=\mathfrak{a}_{a}, \gamma_{g+a}=\mathfrak{b}_{a}$, $a=1, \ldots, g$, are canonically paired, $\left\langle\mathfrak{a}_{a}, \mathfrak{b}_{b}\right\rangle=\delta_{a b}$, and the symplectic form is $J_{a b}=\left\langle\gamma_{a}, \gamma_{b}\right\rangle$. Let $\alpha_{b}$ denote a basis of the harmonic forms paired with the homology cycles $\gamma_{a}$ by $\int_{\gamma_{a}} \alpha_{b}=\delta_{a b}$. With the metric on (complexified as necessary) one-forms $(\alpha, \beta)=\int_{\mathcal{C}} \alpha \wedge * \bar{\beta}$ then we also have that

$$
J_{a b}=\left(* \alpha_{a}, \alpha_{b}\right)=-\left(\alpha_{a}, * \alpha_{b}\right)=\int_{\mathcal{C}} \alpha_{a} \wedge \alpha_{b},
$$


where $*$ is the Hodge star operator.

If $u, v \in H^{1}\left(\mathcal{C}^{\prime}, \mathbb{R}\right)$ then $|G|(u, * v)=\left(\pi^{*} u, * \pi^{*} v\right)$. Letting $\left\{\gamma_{i}^{\prime}\right\}_{i=1}^{2 g^{\prime}},\left\{\alpha_{i}^{\prime}\right\}_{i=1}^{2 g^{\prime}}$ denote the analogous quantities for $\mathrm{C}^{\prime}$ we may write $u=\sum_{i} u_{i} \alpha_{i}^{\prime}$ and similarly for $v$.

Suppose that $p>0$. Then (upon possible relabelling) we have $S\left(\mathfrak{a}_{1}\right)=\mathfrak{a}_{1}, S\left(\mathfrak{b}_{1}\right)=$ $\mathfrak{b}_{1}$ and $S^{*} \alpha_{1}=\alpha_{1}, S^{*} \alpha_{g+1}=\alpha_{g+1}$. Now $\pi^{*} u$ for $u \in H^{1}\left(\mathfrak{C}^{\prime}, \mathbb{R}\right)$ span the invariant differentials of $H^{1}(\mathcal{C}, \mathbb{R})$ and we may find $u, v$ such that $\pi^{*} u=\alpha_{1}, \pi^{*} v=\alpha_{g+1}$. We have that

$$
2(u, * v)=\operatorname{ord}(S)(u, * v)=\left(\pi^{*} u, * \pi^{*} v\right)=\left(\alpha_{1}, * \alpha_{g+1}\right)=-1 .
$$

Now suppose that in addition there exists a fixed point $P$ of $S$. Thus for all $Q$,

$$
\int_{P}^{Q} \alpha_{1}=\int_{P}^{Q} \pi^{*} \alpha_{1}=\int_{P}^{S(Q)} \alpha_{1}
$$

and so consequently (as the path between $Q$ and $S(Q)$ may be arbitrary) $\int_{Q}^{S(Q)} \alpha_{1} \in \mathbb{Z}$. But now if $\gamma^{\prime}$ is any cycle on $\mathcal{C}^{\prime}$ containing the arbitrary point $\pi(Q)$ this may be lifted to a path in $\mathcal{C}$ beginning at $Q$ and ending at $S^{l}(Q)$ for some $l$ (we may assume this lifted path does not pass through any of the fixed points of $S$ ). Then

$$
\int_{\gamma^{\prime}} u=\int_{Q}^{S^{l}(Q)} \pi^{*} u=\int_{Q}^{S^{l}(Q)} \alpha_{1} \in \mathbb{Z}
$$

As this is true for any path $\gamma^{\prime}$ we have $u=\sum_{i} n_{i} \alpha_{i}^{\prime}$ with $n_{i} \in \mathbb{Z}$ and similarly for $v=\sum_{i} m_{i} \alpha_{i}^{\prime}$ with $m_{i} \in \mathbb{Z}$. Therefore $(u, * v)=-n^{T} J m \in \mathbb{Z}$, but from (3) this is not possible. Thus if $p>0$ then $k=0$.

Open Access This article is distributed under the terms of the Creative Commons Attribution 4.0 International License (http://creativecommons.org/licenses/by/4.0/), which permits unrestricted use, distribution, and reproduction in any medium, provided you give appropriate credit to the original author(s) and the source, provide a link to the Creative Commons license, and indicate if changes were made.

\section{References}

1. Accola, R.D.M.: Automorphisms of Riemann surfaces. J. Analyse Math. 18, 1-5 (1967)

2. Braden, H.W., Northover, T.P.: Klein's curve. J. Phys. A 43, \#434009 (2010)

3. Braden, H.W., Northover, T.P.: Bring's curve: its period matrix and the vector of Riemann constants. SIGMA Symmetry Integrability Geom. Methods Appl. 8, \# 065 (2012)

4. Ciliberto, C., Pedrini, C.: Real abelian varieties and real algebraic curves. In: Broglia, F. (ed.) Lectures in Real Geometry. De Gruyter Expositions in Mathematics, vol. 23, pp. 167-256. de Gruyter, Berlin (1996)

5. Comessatti, A.: Sulle varietà abeliane reali. Ann. Mat. Pura Appl. 2(1), 67-106 (1925)

6. Comessatti, A.: Sulle varietà abeliane reali. Ann. Mat. Pura Appl. 3(1), 27-71 (1926)

7. Curtis, C.W., Reiner, I.: Representation Theory of Finite Groups and Associative Algebras. Pure and Applied Mathematics, vol. 10. Interscience, New York (1962)

8. Edge, W.L.: The identification of Klein's quartic. Proc. R. Soc. Edinburgh Sect. A. 62, 83-91 (1944) 
9. Edge, W.L.: A canonical curve of genus 7. Proc. London Math. Soc. 17, 207-225 (1967)

10. Edge, W.L.: Bring's curve. J. London Math. Soc. 18(3), 539-545 (1978)

11. Fay, J.D.: Theta Functions on Riemann Surfaces. Lectures Notes in Mathematics, vol. 352. Springer, Berlin (1973)

12. Gilman, J.: Compact Riemann surfaces with conformal involutions. Proc. Amer. Math. Soc. 37(1), 105-107 (1973)

13. Gilman, J.: A matrix representation for automorphisms of compact Riemann surfaces. Linear Algebra Appl. 17(2), 139-147 (1977)

14. Kalla, C., Klein, C.: Computation of the topological type of a real Riemann surface. Math. Comput. 83(288), 1823-1846 (2014)

15. Newman, M.: Integral Matrices. Pure and Applied Mathematics, vol. 45. Academic Press, New York (1972)

16. Reiner, I.: Symplectic modular complements. Trans. Amer. Math. Soc. 77(3), 498-505 (1954)

17. Riera, G., Rodríguez, R.E.: The period matrix of Bring's curve. Pacific J. Math. 154(1), 179-200 (1992)

18. Silhol, R.: Real abelian varieties and the theory of Comessatti. Math. Z. 181(3), 345-364 (1982)

19. Vinnikov, V.: Selfadjoint determinantal representations of real plane curves. Math. Ann. 296(3), 453479 (1993) 